\title{
MicroRNA-216a enhances the radiosensitivity of pancreatic cancer cells by inhibiting beclin-1-mediated autophagy
}

\author{
XIANGLIANG ZHANG $^{1 *}$, HUIJUAN SHI $^{2 *}$, SHENGQV LIN $^{1}$, MINGCHEN BA $^{1}$ and SHUZHONG CUI ${ }^{1}$ \\ ${ }^{1}$ Department of Abdominal Surgery (Section 2), The Affiliated Cancer Hospital of Guangzhou Medical University, \\ Guangzhou, Guangdong 510095; ${ }^{2}$ Department of Pathology, The First Affiliated Hospital of Sun Yat-Sen University, \\ Guangzhou, Guangdong 510080, P.R. China
}

Received March 9, 2015; Accepted June 11, 2015

DOI: $10.3892 /$ or.2015.4078

\begin{abstract}
Radioresistance has become a challenge in the treatment of pancreatic cancer, which limits the efficacy and outcomes of radiotherapy in clinical treatment. Autophagy, recognized as an adaptive response to cell stress, has recently been involved in the radioresistance of cancer cells. MicroRNAs (miRNAs) are also involved in the radioresistance of pancreatic cancer cells. In the present study, we established a radioresistant pancreatic cancer cell line and found that miRNA-216a was significantly downregulated whereas the autophagy activity was increased as compared with the control. Forced expression of miR-216a was found to inhibit the expression of beclin-1, a critical autophagic gene, as well as autophagy. Using bioinformatics analysis and the dual-luciferase reporter gene assay, we found that miR-216a directly interacted with 3'-untranslated region (UTR) of beclin-1. Furthermore, the forced expression of miR-216a inhibited cell growth and colony formation ability and promoted the cell apoptosis of radioresistant pancreatic cancer cells in response to irradiation. By contrast, overexpression of beclin-1 abrogated the effects of miR-216a. Furthermore, miR-216a sensitized xenograft tumor to irradiation treatment and inhibited irradiation-induced autophagy by regulating beclin-1. Collectively, the results demonstrated that miR-216a enhanced the radiosensitivity of pancreatic cancer
\end{abstract}

Correspondence to: Professor Shuzhong Cui, Department of Abdominal Surgery (Section 2), The Affiliated Cancer Hospital of Guangzhou Medical University, 78 Hengzhigang Road, Guangzhou, Guangdong 510095, P.R. China

E-mail: 513340728@qq.com

*Contributed equally

Abbreviations: miRNAs, microRNAs; UTR, untranslated region; LC3, microtubule-associated protein light chain 3; MTT, 3-(4,5dimethylthiazol-2-yl)-2,5-diphenyl-tetrazolium bromide; TUNEL, terminal deoxynucleotidyl transferase dUTP nick end-labeling

Key words: pancreatic cancer, microRNA, autophagy, radioresistance cells by inhibiting beclin-1-mediated autophagy, suggesting a promising molecular target for improving the radiotherapy of pancreatic cancer.

\section{Introduction}

Pancreatic cancer, one of the most lethal types of cancer, lacks effective therapeutic methods and exhibits a low 5-year survival rate of $<5 \%$ (1). Although surgical resection is the most important and curative treatment methods for cancer, pancreatic cancer is usually unresectable at the time of diagnosis $(2,3)$. Therefore, radiotherapy alone or as an adjuvant treatment has been used as major therapeutic methods for pancreatic cancer (4). However, the radioresistance of pancreatic cancer severely affects the efficacy and outcomes of radiotherapy in clinical treatment $(5,6)$. Accordingly, overcoming radioresistance has become crucial in cancer radiotherapy in recent years (7).

Autophagy, a catabolic process for the degradation of cytoplasmic proteins and organelles as an adaptive response to cell stress such as nutrient starvation or metabolic stress, is considered a potential mechanism for the radioresistance of cancer cells (8). Autophagy is a membrane trafficking process in which the autophagosome formation is triggered by class III phosphoinositide 3-kinase and beclin-1 (also known as the mammalian homologue of the yeast autophagy-related gene 6) (9). Beclin-1 is a critical gene for autophagosome formation that shows high expression levels during autophagy (10). Conversion of the microtubule-associated protein light chain 3 (LC3) from LC3-I (cytosolic form) to LC3-II (autophagic membrane form) is another critical process during autophagy and the levels of LC3I/II have been considered a classic marker for the detection of autophagy (11). Mounting evidence has demonstrated that inhibition of autophagy by 3-methyladenine and chloroquine effectively enhanced the radiosensitivity of cancer cells (12-14). Therefore, targeting autophagy to inhibit radioresistance of pancreatic cancer cells is a promising research direction for improving clinical outcomes.

MicroRNAs (miRNAs), the post transcriptional regulators of gene expression, have been identified as an important regulator in a variety of cell processes (15). They are 18-24 nucleotides in length and bind to the 3'-untranslated region (UTR) of the target mRNA leading to mRNA destabilization and thereby 
protein translational inhibition $(16,17)$. The role of miRNAs in tumorigenesis and cancer treatment has been well characterized. miRNAs are associated with patient survival and are useful predictors and modificators for anticancer treatment (18-20). However, the potential underlying mechanisms of miRNAs in regulating radioresistance of pancreatic cancer cells remains largely unknown.

Previous findings have indicated that miR-216a was markedly decreased in pancreatic cancer (21-23), suggesting an important role of miR-216a in pancreatic cancer. In the present study, using bioinformatic algorithms we found that beclin-1, an important regulator of autophagy, was a putative target gene of miR-216a. Consequently, we investigated whether miR-216a targeted beclin-1-mediated autophagy and played a critical role in the radioresistance of pancreatic cancer cells. In the present study, we found that miR-216a was inhibited in radioresistant pancreatic cancer cells and that beclin-1 as well as autophagy activity were highly upregulated. We also found that forced expression of miR-216a significantly suppressed beclin-1 and autophagy activity in radioresistant pancreatic cancer cells, which enhanced the radiosensitivity of pancreatic cancer cells. Thus, miR-216a is a promising target that can be used to sensitize pancreatic cancer cells to irradiation by abrogating irradiation-induced autophagy.

\section{Materials and methods}

Cell culture and mice. The human pancreatic cancer cell line, PANC-1, was obtained from the Chinese Academy of Sciences (Shanghai, China) and cultured in Dulbecco's modified Eagle's medium (DMEM; Invitrogen-Life Technologies, Carlsbad, CA, USA) supplemented with $10 \%$ fetal bovine serum (Invitrogen-Life Technologies) containing penicillin/ streptomycin. The cells were grown in a humidified $5 \% \mathrm{CO}_{2}$ at $37^{\circ} \mathrm{C}$ in an incubator. The irradiation of PANC-1 cells was performed according to a previously reported method (24). PANC-1 cells grown in complete medium were subjected to $2 \mathrm{~Gy}{ }^{60} \mathrm{Co}$ radiation at $2 \mathrm{~Gy} / \mathrm{min}$ using an X-ray machine (X-RAD 320, Precision X-ray) at the Institute of Radiation Medicine of Affiliated Cancer Hospital of Guangzhou Medical University (Guangdong, China). The irradiated cells were then sub-cultured in new plates and irradiated with increasing doses of irradiation $(4,6,8$, and $10 \mathrm{~Gy})$ for subsequent experiment.

Female 6-week-old BALB/c nude mice (25-30 g) were obtained from the Medical Experimental Animal Center (Guangdong, China) and housed under pathogen-free conditions with free access to water and food. The animal experimental procedures were approved and reviewed by the Institutional Animal Care and Use Committee of Guangzhou Medical University.

Cell viability and colony formation assays. Following irradiation or miR-216a treatment, cell growth and viability was evaluated using a 3-(4,5-dimethylthiazol-2-yl)-2,5-diphenyltetrazolium bromide (MTT; Sangon, Shanghai, China) assay. Briefly, the cells were seeded in 96-well plates at a density of $5 \times 10^{3}$ cells $/ 200 \mu \mathrm{l}$. After treatment, fresh medium containing MTT $[5 \mathrm{mg} / \mathrm{ml}$ diluted in phosphate-buffered saline (PBS), $20 \mu \mathrm{l} /$ well] was added and incubated for an additional $4 \mathrm{~h}$. The formed formazan was resuspended with dimethyl sulfoxide
(200 $\mu \mathrm{l} /$ well). Absorbance was determined at $490 \mathrm{~nm}$ using an ELISA reader (Bio-Tek, Winooski, VT, USA). For detection of the colony formation ability, the cells following treatment were grown in 6-well plates and cultured for 15 days. The old medium was discarded and cell colonies stained with crystal violet were counted using a dissecting microscope. The experiments were performed in quadruplicate and repeated three times.

TUNEL assay. Apoptotic cells were stained using the TUNEL (terminal deoxynucleotidyl transferase dUTP nick end-labeling) apoptosis kit (Genmed Scientifics, Arlington, MA, USA) according to the supplier's instructions. Briefly, the cells were fixed with $4 \%$ paraformaldehyde followed by incubation with TUNEL reaction mixtures for $1 \mathrm{~h}$ at $37^{\circ} \mathrm{C}$. The stained cells were visualized and counted using a fluorescence microscope (Olympus, Tokyo, Japan). Five fields (magnification, $\mathrm{x} 400$ ) were randomly selected for the measurement of apoptotic cells in a blinded manner.

Dual-luciferase reporter assay. The beclin-1 3'-UTR and mutated 3'-UTR constructs were amplified and subcloned into pGL3 Luciferase Promoter Vector (Promega, Madison, WI, USA) with $X b a \mathrm{I}$ and NotI restriction sites. Using Lipofectamine transfection reagent (Invitrogen-Life Technologies) the pGL3 vector containing beclin-1 3'-UTR or mutated forms was co-transfected with or without $20 \mathrm{nmol} / 1 \mathrm{miR}-216 \mathrm{a} \mathrm{mimic}$ (GenePharma, Shanghai, China) into PANC-1 cells according to the manufacturer's instructions. The cells were collected after 48-h transfection and the luciferase activity was measured using the Dual-Luciferase Reporter Assay kit (Promega). The relative protein expression levels of beclin-1 and LC3-II were quantified using Image-Pro Plus 6.0 software. The relative luciferase activities were normalized with GAPDH to that of the control cells.

Reverse transcriptase-quantitative polymerase chain reaction $(R T-q P C R)$. Total RNA was isolated by using TRIzol (Invitrogen-Life Technologies) and small RNAs were extracted by using mirVana kits (Ambion Inc, Austin, TX, USA) according to the manufacturer's instructions. Corresponding cDNA was generated by using M-MLV reverse transcriptase (Clontech, Palo Alto, CA, USA) and the TaqMan miRNA Reverse Transcription kit (Applied Biosystems, Foster City, CA, USA) according to the manufacturer's instructions. To analyze the gene expression levels, the RT-qPCR mixture system containing cDNA templates, primers and SYBR-Green qPCR Master Mix were subjected to RT-qPCR quantification. Glyceraldehyde-3-phosphate dehydrogenase (GAPDH; for beclin-1) and U6 SnRNA (for miR-216a) were used as an internal reference and relative gene expression was quantified by $2^{-\Delta \Delta \mathrm{Ct}}$ method.

Western blot analysis. Total cell lysates were separated by $10 \%$ sodium dodecyl sulfate-polyacrylamide gel electrophoresis and transferred to nitrocellulose membranes (Amersham, Little Chalfont, UK). Non-fat dry milk (2.5\%) was used for blocking the membrane and primary antibodies including anti-LC3 antibody (ab63817) and anti-beclin-1 (ab62557) (both from Abcam, Cambridge, UK), anti-cleaved caspase-3 
A

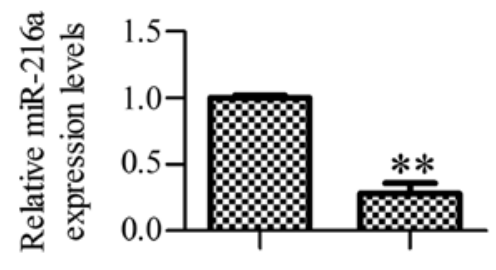

C

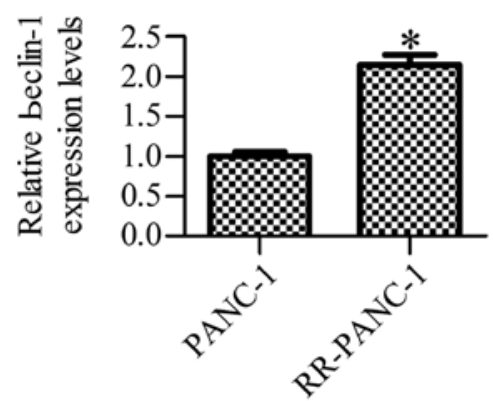

B

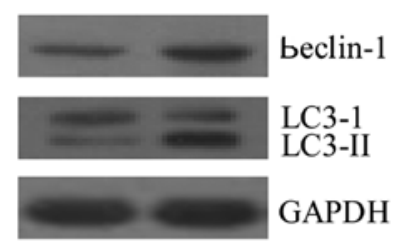

D

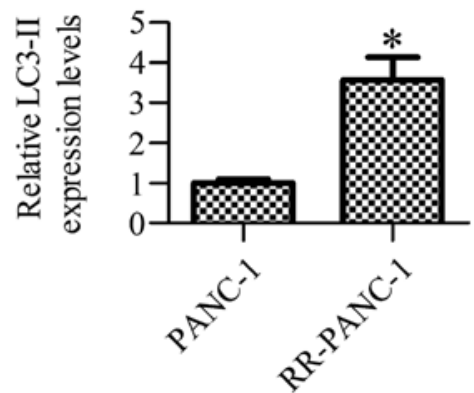

Figure 1. Effect of irradiation on miR-216a expression and autophagy activity in PANC-1 cells. (A) qRT-PCR was performed to analyze the expression levels of miR-216a in RR-PANC-1 cells. (B) Western blot analysis was performed to detect the autophagy activity indicated by beclin-1 and LC3 I/II protein expression levels. Relative protein expression levels of beclin-1 (C) and LC3-II (D) were quantified using Image-Pro Plus 6.0 software and normalized to GAPDH. PANC-1 denotes PANC-1 cells without irradiation, RR-PANC-1 denotes radioresistant PANC-1 cells. ${ }^{*}<0.05$ and ${ }^{* *} \mathrm{P}<0.01$. PANC-1, human pancreatic cancer cell line; RT-qPCR, reverse transcriptase-quantitative polymerase chain reaction; LC3, microtubule-associated protein light chain 3.

(PC679-50UG) (from Millipore, Boston, MA, USA) and antiGAPDH antibody (bs-2188R) (Bioss, Beijing, China) were used for detection of the target protein. The target protein was visualized by using an enhanced chemiluminescence (ECL) detection system (Amersham).

Tumorigenicity assay. Cells $\left(2 \times 10^{6}\right)$ diluted in $200 \mu \mathrm{l}$ PBS were injected subcutaneously into the right groin of $\mathrm{BALB} / \mathrm{c}$ nude mice. The tumor volume was measured daily and the tumor was irradiated with a dose of irradiation (10 Gy) when the volume reached $\sim 500 \mathrm{~mm}^{3}$. The length and width were measured and the volume was calculated using the formula: length $\mathrm{x}$ width ${ }^{2} \mathrm{x} \pi / 6$.

Statistical analysis. Data were presented as the mean \pm standard deviation (SD) of three or more independent experiments. Levels of significance between or among groups were analyzed by the two-tailed Student's t-test or the one-way ANOVA, respectively. Results were considered statistically significant at $\mathrm{P}<0.05$.

\section{Results}

Irradiation induces miR-216a inhibition and autophagy activation in PANC-1 cells. To investigate the potential role of miR-216a in radioresistance of pancreatic cancer cells, we first examined the miR-216a levels in radioresistant cells using RT-qPCR. The results showed that miR-216a expression levels were significantly inhibited in radioresistant (RR)-PANC-1 cells as compared with the control PANC-1 cells (Fig. 1A). We also observed an increased level of autophagy activity as indicated by upregulation of the beclin-1 and LC3-II protein levels in RR-PANC-1 cells in comparison with control cells as determined by western blot analysis (Fig. 1B-D). The data suggested that irradiation contributed to miR-216a inhibition and autophagy activation.

Forced expression of miR-216a inhibits the irradiationinduced upregulation of beclin-1 and autophagy activity. To investigate whether the inhibited miR-216a expression has a certain correlation with the increased autophagy activity, we treated the RR-PANC-1 cells with miR-216a mimics and determined the alterations of autophagy activity. The results exhibited that forced expression of miR-216a significantly suppressed beclin-1 and LC3-II protein expression levels (Fig. 2A and B), suggesting that miR-216a played an important role in the regulation of autophagy activity. Fig. $2 \mathrm{C}$ shows that forced expression of miR-216a had suppressed LC3-II protein expression levels significantly too.

miR-216a directly targets beclin-1 to regulate autophagy activity. To investigate the relationship between miR-216a and autophagy, we screened whether miR-216a has a direct interaction with autophagy-related genes using bioinformatics analysis. As expected, we found that beclin-1 was a putative target gene of miR-216a (Fig. 3A). To determine whether beclin-1 3'-UTR was responsive to miR-216a, we performed the dual-luciferase reporter assay by using pGL3-beclin-1-3'-UTR and miR-216a mimics. Co-transfection of pGL3-beclin-13'-UTR with miR-216a mimics in PANC-1 cells significantly downregulated the relative luciferase activity in comparison with cells co-transfected with miR-216a, with pGL3-beclin-1mut-3'-UTR containing a mutation in the predicted consensus sequences for miR-216a (Fig. 3B). These findings suggested that beclin-1 is a direct target gene of miR-216a.

Forced expression of miR-216a reduces cell growth and colony formation in RR-PANC-1 cells in response to irradiation. To 
A

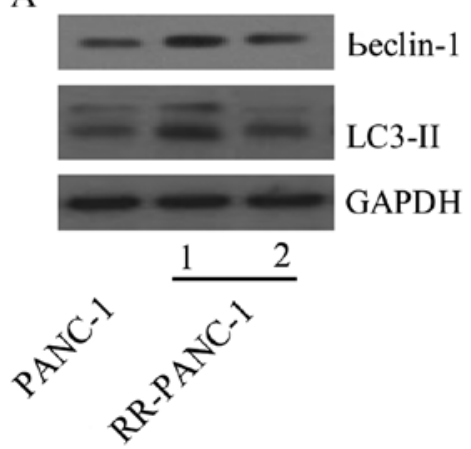

B

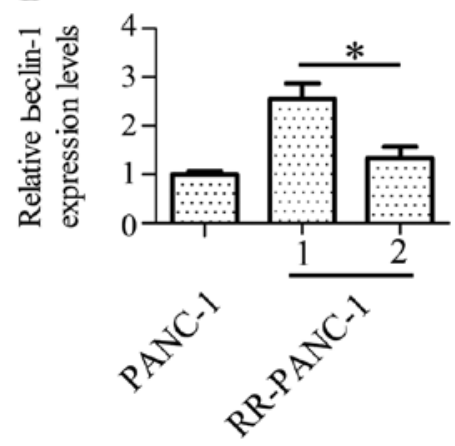

$\mathrm{C}$

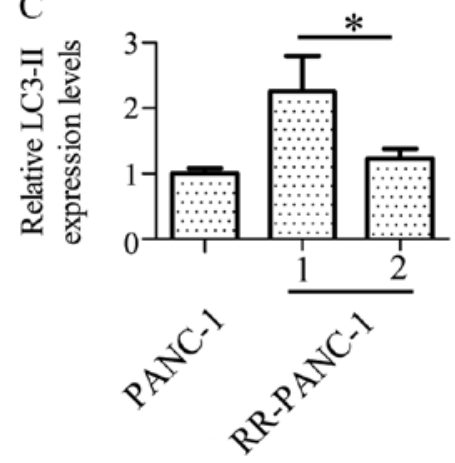

Figure 2. Effect of miR-216a on autophagy in RR-PANC-1 cells. (A) Beclin-1 and LC3 I/II protein expression was detected by western blot analysis using the indicated antibodies. Relative protein expression levels of (B) beclin-1 and (C) LC3-II were quantified using Image-Pro Plus 6.0 software and normalized to GAPDH * $\mathrm{P}<0.05$. PANC-1, human pancreatic cancer cell line; LC3, microtubule-associated protein light chain 3.

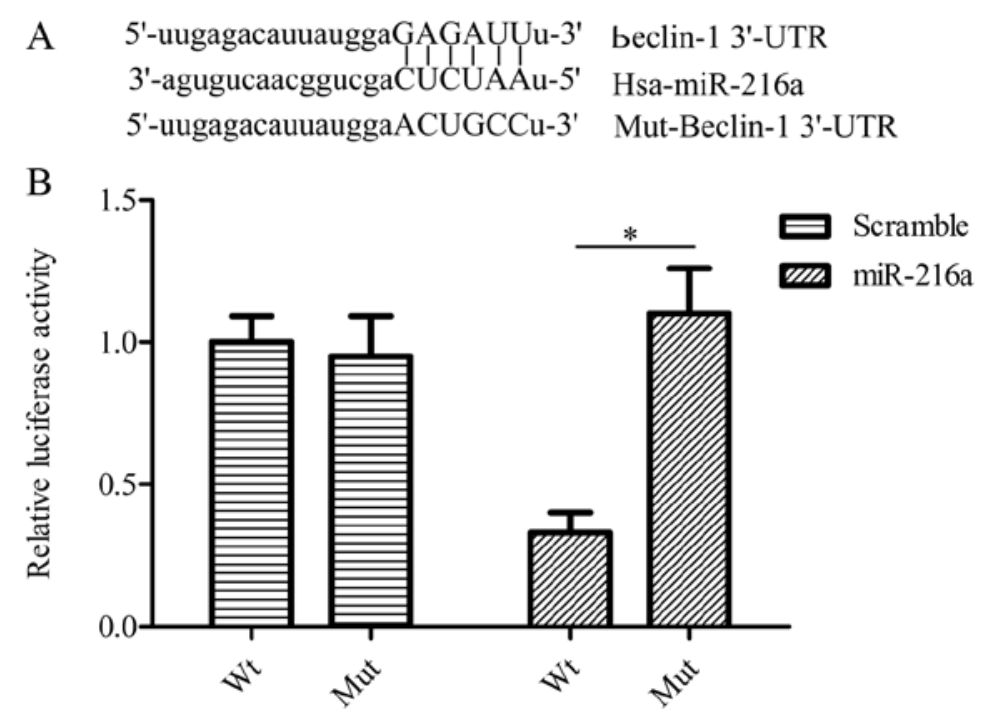

Figure 3. miR-216a directly targets 3'-UTR of beclin-1. (A) The predicted binding sequences for beclin-1 3'-UTR with miR-216a. (B) Luciferase activity assays were used to detect the interaction between miR-216a and beclin-1 3'-UTR. The wild-type or mutant beclin-1 3'-UTR was co-transfected with miR-216a mimics or scrambled miRNA and incubated for $48 \mathrm{~h}$ in PANC-1 cells followed using a detection by dual-luciferase reporter assay kit. Wt, pGL3 vectors containing beclin-1 3'-UTR; Mut, pG3 vectors containing mutated Beclin-1 3'-UTR as mentioned above. P<0.05. PANC-1, human pancreatic cancer cell line; UTR, 3'-untranslated region.

assess whether miR-216a-mediated autophagy inhibition via beclin-1 plays an important role in the growth of RR cells in response to irradiation, we forced the expression of miR-216a in RR cells and analyzed the cell growth and colony formation after irradiation. RR-PANC-1 cells were pretreated with miR-216a mimics or control scramble miRNA for $1 \mathrm{~h}$ and then subjected to irradiation ( $2 \mathrm{~Gy} / \mathrm{min}$ ). Using the MTT assay, we found that the forced expression of miR-216a markedly sensitized RR cells to cell death in response to irradiation, as compared with the control group (Fig. 4A). Furthermore, the colony formation ability of RR-PANC-1 cells was inhibited by miR-216a (Fig. 4B). These data suggested that miR-216a sensitized RR-PANC-1 cells to irradiation.

Forced expression of miR-216a sensitizes RR-PANC-1 cells to irradiation by elevating cell apoptosis. To study the role of miR-216a on RR cells, we detected the effect of miR-216a on cell apoptosis in RR cells in response to irradiation. The results of the TUNEL assay indicated that a forced expression of miR- 216a significantly increased the cell apoptosis of RR-PANC-1 cells in comparison with the control (Fig. 5A). In addition, the pro-apoptotic protein expression level of cleaved caspase-3 was also increased by miR-216a treatment (Fig. 5B and C). Collectively, these results suggested that miR-216a enhanced the radiosensitivity of RR-PANC-1 cells via activation of cell apoptosis.

Overexpression of beclin-1 abrogates miR-216a-induced irradiation sensitivity. To verify whether miR-216a sensitized RR-PANC-1 cells to irradiation by regulating beclin-1, we co-transfected miR-216a with beclin-1 overexpression vectors harboring no specific miR-216a binding specific sequences in 3'-UTR in RR-PANC-1 cells. The results showed that overexpression of beclin-1 (Fig. 6A and B) significantly blocked miR-216a-induced cell growth inhibition in RR-PANC-1cells in response to irradiation detected by the MTT assay (Fig. 6C). Furthermore, overexpression of beclin-1 abrogated cell apoptosis induced by miR-216a in RR-PANC-1 cells as indicated 


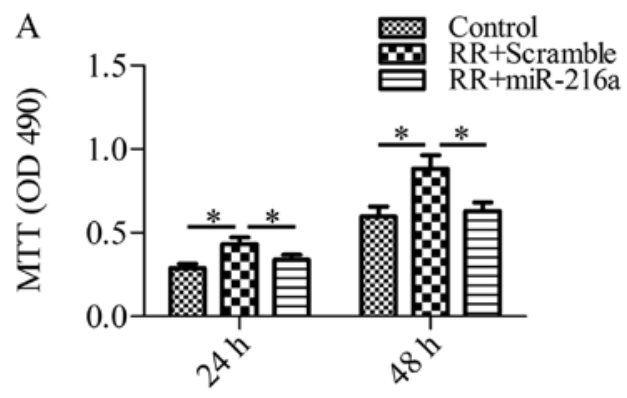

B

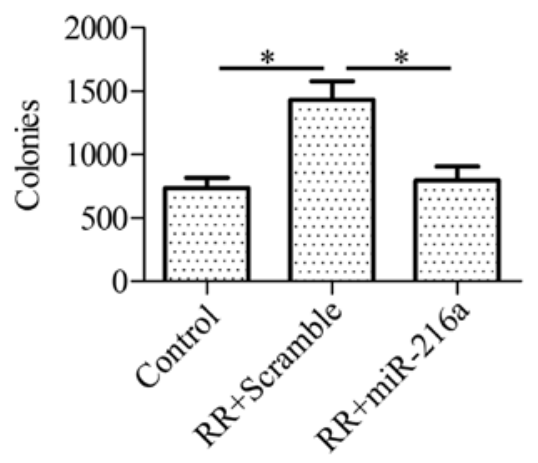

Figure 4. Effect of miR-216a on cell growth and colony formation in RR-PANC-1 cells. (A) Cell growth was detected by MTT assay. Control PANC-1 cells, or RR-PANC-1 cells pretreated with control scramble miRNA and miR-216a mimics were irradiated ( $2 \mathrm{~Gy} / \mathrm{min})$. After 24 and $48 \mathrm{~h}$ treatment, cell growth was determined. (B) Cells subjected to irradiation were continued to be cultured for 15 days. Crystal violet staining was performed and the cell colonies were counted. ${ }^{\mathrm{P}}<0.05$. PANC-1, human pancreatic cancer cell line.
A

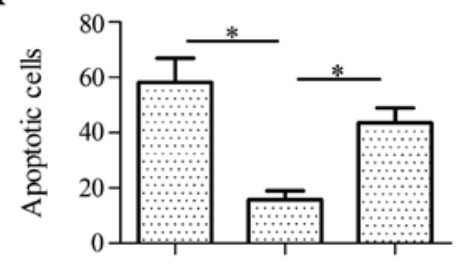

B

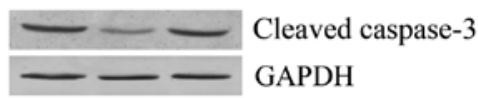

$\mathrm{C}$

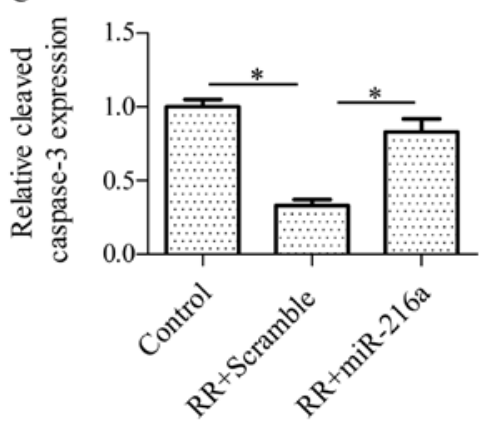

Figure 5. Effect of miR-216a on cells apoptosis in RR-PANC-1 cells. (A) Apoptotic cells were detected by TUNEL assay. Control PANC-1 cells or RR-PANC-1 cells pretreated with control scramble miRNA and miR-216a mimics were irradiated $(2 \mathrm{~Gy} / \mathrm{min})$ and cultured for $48 \mathrm{~h}$. Apoptotic cells were counted in a field of 500x $500 \mu \mathrm{m}$. (B) The cleaved caspase- 3 in cell lysates was detected by western blot analysis. (C) Relative protein expression was quantified using Image-Pro Plus 6.0 software and normalized to GAPDH. "P<0.05. PANC-1, human pancreatic cancer cell line.

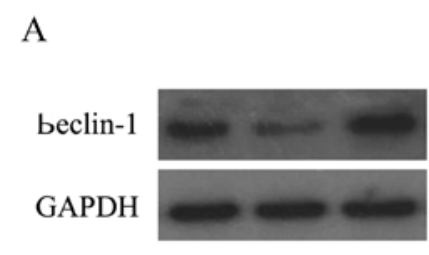

B

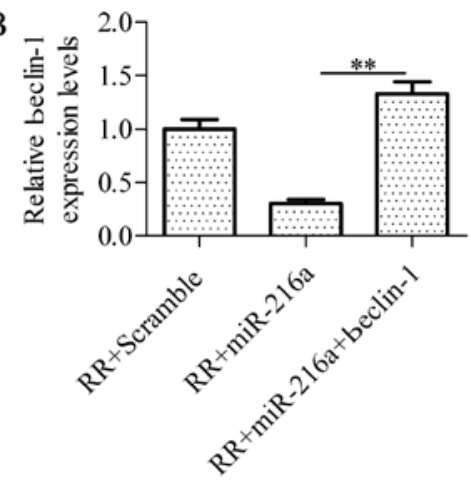

C

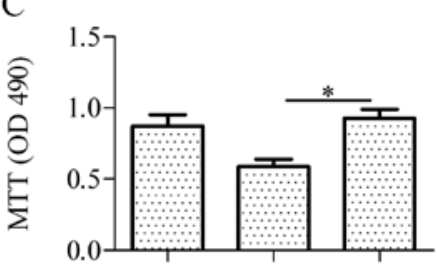

D

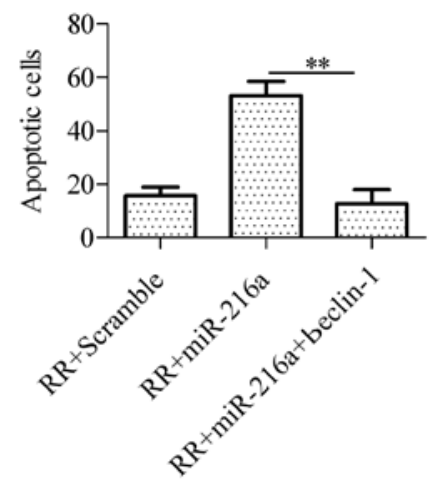

Figure 6. Effect of beclin-1 overexpression on miR-216a-induced irradiation sensitivity in RR-PANC-1 cells. (A) Western blot analysis to detect the expression levels of beclin-1 in different treated groups. RR-PANC-1 cells treated with miR-216a with or without beclin-1 overexpression vectors were subjected to irradiation $(2 \mathrm{~Gy} / \mathrm{min})$ and cultured for $48 \mathrm{~h}$. Cells treated with scramble oligonucleotides were regarded as the control. (B) Relative protein expression was quantified using Image-Pro Plus 6.0 software and normalized to GAPDH. Cell growth (C) and apoptosis (D) was detected by MTT assay and TUNEL assay, respectively. ${ }^{*} \mathrm{P}<0.05$ and ${ }^{* *} \mathrm{P}<0.01$. PANC-1, human pancreatic cancer cell line. 
A
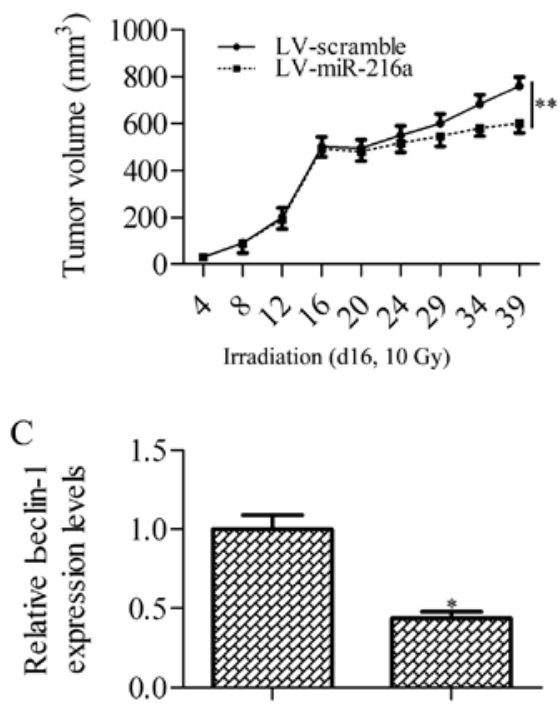

B

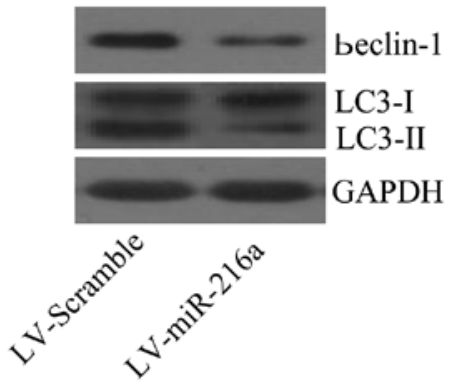

$\mathrm{D}$

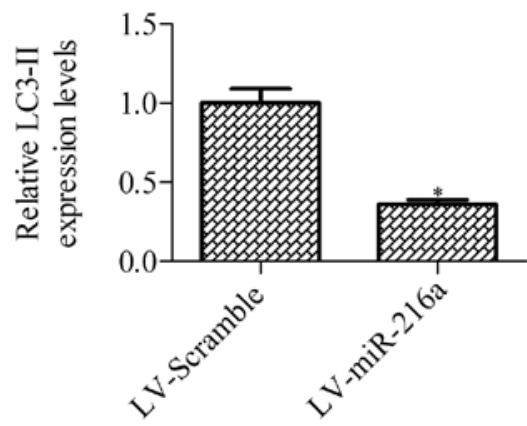

Figure 7. Effect of miR-216a on xenograft tumor growth in vivo. (A) Nude mice were subcutaneously injected with PANC-1 cells infected with control lentiviral vector or miR-216a overexpressing lentiviral vector. The tumors were irradiated (10 Gy) on day 16 when the average tumor volume reached $\sim 500 \mathrm{~mm}^{3}$. (B) Protein expression levels in xenograft samples were detected by western blot analysis with the indicated antibodies. Relative protein expression levels of (C) beclin-1 and (D) LC3-II were quantified using Image-Pro Plus 6.0 software and normalized to GAPDH. * $<0.05$. PANC-1, human pancreatic cancer cell line; LC3, microtubule-associated protein light chain 3.

by the TUNEL assay (Fig. 6D). These findings suggested that beclin-1 is important in regulating the irradiation sensitivity in RR cells, further confirming that miR-216a targeted beclin-1 to sensitized RR-PANC-1 cells to irradiation.

miR-216a sensitizes pancreatic cancer cells to irradiation treatment by inhibiting irradiation-induced autophagy in xenograft tumor. To assess whether miR-216a can increase the efficiency of irradiation in killing implanted tumors in nude mice, nude mice were subcutaneously injected with PANC-1 cells pre-transfected with lentiviral vector expressing miR-216a or scramble miRNA control. When the tumors reached $\sim 500 \mathrm{~mm}^{3}$ on day 16 , the tumors received a single dose of 10-Gy irradiation. The results showed that force expression of miR-216a significantly increased the radiosensitivity of PANC-1-derived tumors on irradiation treatment (Fig. 7A). We further analyzed beclin-1 expression and autophagy activity in the xenograft samples after irradiation treatment. Consistent with the above results, miR-216a inhibited irradiation-induced beclin-1 expression and autophagy activity (Fig. 7B-D). These results demonstrated that miR-216a sensitizes pancreatic cancer cells to irradiation treatment by blocking irradiationinduced beclin-1-mediated autophagy.

\section{Discussion}

The role of miR-216a in cancer has increasingly drawn attention. The transcription of miR-216a has been found to be stimulated by the androgen pathway which targets the tumor suppressor in lung cancer-1 gene in the early stage of carcinogenesis (25). miR-216a targets phosphatase and tensin homolog and decapentaplegic homolog 7 to induce mesen- chymal transition in hepatocellular carcinoma (26). miR-216a seems to act as an oncogene in hepatocellular carcinoma. However, miR-216a was reported to be downregulated in pancreatic cancer $(21,22)$. The downregulation of miR-216a in feces has been suggested as a biomarkers for pancreatic cancer (23). In pancreatic intraepithelial neoplasms, miR-216a was also found to be decreased (27). The suppressed expression patterns of miR-216a in pancreatic cancer suggests that miR-216a plays an important role in the tumorigenesis of pancreatic cancers and is a promising molecular target for the treatment of pancreatic cancer.

As expected, we found that miR-216a was involved in the regulation of radioresistance through beclin-1-mediated autophagy. Autophagy, an adaptive response against cellular streak, has been currently suggested to be involved in the radioresistance of cancer cells (8). However, the role of autophagy in the radioresistance of cancer cells remains controversial. $\gamma$-radiation induces autophagy, which is responsible for the radioresistance of glioma stem cells $(28,29)$. In breast cancer, inhibition of autophagy promoted radiosensitivity via the suppression of transforming growth factor-activated kinase-1 (8). Hypoxia-induced autophagy was suggested to attribute to the radioresistance of breast cancer cells (30). Chaachouay et al have demonstrated that radioresistant breast cancer cells exhibit a high level of autophagy serving as a protective and survival mechanism against irradiation (31). Anticancer drugs, Akt inhibitors, were revealed to promote radiosensitivity through induction of autophagy (32). Similarly, the inhibitors of the mammalian target of rapamycin radiosensitized non-small cell lung cancer cells harboring phosphatase and tensin homolog deficient and epidermal growth factor receptor activating mutant (33). The 
apparent dual role of autophagy in the irradiation of cancer cells remains elusive, suggesting that autophagy has different biological functions due to the different cell type or stimuli.

The role of autophagy in the regulation of radioresistance of pancreatic cancer has been investigated. Profilin1 has been indicated to be capable of sensitizing pancreatic cancer cells to irradiation by inhibiting autophagy (34). More recently, the overexpression of miR-23b has been shown to decrease radiation-induced autophagy and increase the radiosensitivity of pancreatic cancer cells (35). Consistently, the inhibition of autophagy by miR-216a was found to enhance the radiosensitivity of pancreatic cancer cells in our study. We have demonstrated that miR-216a was significantly downregulated by irradiation and the autophagy activity was increased in pancreatic cancer cells in response to irradiation, suggesting a potential association between autophagy and miR-216a. As expected, we further identified and characterized that miR-216a targeted a critical autophagic gene, beclin-1, to inhibit autophagy activation in response to irradiation in pancreatic cancer cells. Consistent with our findings, Menghini et al have demonstrated that miR-216a targets beclin-1 to regulate autophagy in endothelial cells contributing to the endothelial function in cardiovascular diseases (36).

miR-30a sensitizes cancer cells to cis-platinum via suppression of beclin 1-mediated autophagy (37). The downregulation of miR-17-5p-induced beclin-1 overexpression leads to paclitaxel resistance in lung cancer cells (38). beclin-1 has been suggested to be important in the chemoradiation which affects the overall survival of patients with esophageal squamous cell carcinoma (39). Therefore, considering the role of beclin-1 in anticancer therapy, targeting beclin-1 may have a better outcome in the treatment of pancreatic cancer. In conclusion, our results provide evidence that miR-216a inhibited beclin-1 leading to the downregulation of autophagy induced by irradiation, which enhanced the radiosensitivity of pancreatic cancer cells.

\section{Acknowledgements}

The present study was supported by the Guangdong Province Natural Science Foundation (S2013010016662), the Health Bureau of Guangdong Province (A2014224 and B2014196), the Science and Technology Planning Project of Guangdong Province (2013B021800284) and the National Natural Science Foundation of China (81201932 and 81372493).

\section{References}

1. Vincent A, Herman J, Schulick R, Hruban RH and Goggins M: Pancreatic cancer. Lancet 378: 607-620, 2011.

2. Siegel R, Naishadham D and Jemal A: Cancer statistics, 2012. CA Cancer J Clin 62: 10-29, 2012.

3. Li D, Xie K, Wolff R and Abbruzzese JL: Pancreatic cancer. Lancet 363: 1049-1057, 2004.

4. Neoptolemos JP, Dunn JA, Stocken DD, Almond J, Link K, Beger H, Bassi C, Falconi M, Pederzoli P, Dervenis C, et al, European Study Group for Pancreatic Cancer: Adjuvant chemoradiotherapy and chemotherapy in resectable pancreatic cancer: A randomised controlled trial. Lancet 358: 1576-1585, 2001.

5. Girard N, Mornex F, Bossard N, Ychou M, Chauffert B and Wautot V: Estimating optimal dose of twice-weekly gemcitabine for concurrent chemoradiotherapy in unresectable pancreatic carcinoma: Mature results of GEMRT-01 Phase I trial. Int J Radiat Oncol Biol Phys 77: 1426-1432, 2010.
6. Crane CH, Abbruzzese JL, Evans DB, Wolff RA, Ballo MT, Delclos M, Milas L, Mason K, Charnsangavej C, Pisters PW, et al: Is the therapeutic index better with gemcitabine-based chemoradiation than with 5-fluorouracil-based chemoradiation in locally advanced pancreatic cancer? Int J Radiat Oncol Biol Phys 52: 1293-1302, 2002.

7. Chatterjee S, Willis N, Locks SM, Mott JH and Kelly CG: Dosimetric and radiobiological comparison of helical tomotherapy, forward-planned intensity-modulated radiotherapy and two-phase conformal plans for radical radiotherapy treatment of head and neck squamous cell carcinomas. Br J Radiol 84: 1083-1090, 2011.

8. Han MW, Lee JC, Choi JY, Kim GC, Chang HW, Nam HY, Kim SW and Kim SY: Autophagy inhibition can overcome radioresistance in breast cancer cells through suppression of TAK1 activation. Anticancer Res 34: 1449-1455, 2014.

9. Kondo Y, Kanzawa T, Sawaya R and Kondo S: The role of autophagy in cancer development and response to therapy. Nat Rev Cancer 5: 726-734, 2005

10. Maiuri MC, Zalckvar E, Kimchi A and Kroemer G: Self-eating and self-killing: Crosstalk between autophagy and apoptosis. Nat Rev Mol Cell Biol 8: 741-752, 2007.

11. Mizushima N, Ohsumi Y and Yoshimori T: Autophagosome formation in mammalian cells. Cell Struct Funct 27: 421-429, 2002.

12. Liang B, Kong D, Liu Y, Liang N, He M, Ma S and Liu X: Autophagy inhibition plays the synergetic killing roles with radiation in the multi-drug resistant SKVCR ovarian cancer cells. Radiat Oncol 7: 213, 2012.

13. Gewirtz DA, Hilliker ML and Wilson EN: Promotion of autophagy as a mechanism for radiation sensitization of breast tumor cells. Radiother Oncol 92: 323-328, 2009.

14. Zois CE and Koukourakis MI: Radiation-induced autophagy in normal and cancer cells: Towards novel cytoprotection and radio-sensitization policies? Autophagy 5: 442-450, 2009.

15. Mendell JT and Olson EN: MicroRNAs in stress signaling and human disease. Cell 148: 1172-1187, 2012.

16. Bartel DP: MicroRNAs: Genomics, biogenesis, mechanism, and function. Cell 116: 281-297, 2004.

17. Winter J, Jung S, Keller S, Gregory RI and Diederichs S: Many roads to maturity: microRNA biogenesis pathways and their regulation. Nat Cell Biol 11: 228-234, 2009.

18. Wang P, Chen L, Zhang J, Chen H, Fan J, Wang K, Luo J, Chen Z, Meng Z and Liu L: Methylation-mediated silencing of the miR-124 genes facilitates pancreatic cancer progression and metastasis by targeting Rac1. Oncogene 33: 514-524, 2014.

19. Oh JS, Kim JJ, Byun JY and Kim IA: Lin28-let7 modulates radiosensitivity of human cancer cells with activation of K-Ras. Int J Radiat Oncol Biol Phys 76: 5-8, 2010.

20. Wang P, Zhuang L, Zhang J, Fan J, Luo J, Chen H, Wang K, Liu L, Chen Z and Meng Z: The serum miR-21 level serves as a predictor for the chemosensitivity of advanced pancreatic cancer, and miR-21 expression confers chemoresistance by targeting FasL. Mol Oncol 7: 334-345, 2013

21. Hou B, Jian Z, Chen S, Ou Y, Li S and Ou J: Expression of miR-216a in pancreatic cancer and its clinical significance. Nan Fang Yi Ke Da Xue Xue Bao 32: 1628-1631, 2012 (In Chinese).

22. Ali S, Banerjee S, Logna F, Bao B, Philip PA, Korc M and Sarkar FH: Inactivation of Ink4a/Arf leads to deregulated expression of miRNAs in K-Ras transgenic mouse model of pancreatic cancer. J Cell Physiol 227: 3373-3380, 2012.

23. Link A, Becker V, Goel A, Wex T and Malfertheiner P: Feasibility of fecal microRNAs as novel biomarkers for pancreatic cancer. PLoS One 7: e42933, 2012.

24. Skvortsov S, Jimenez CR, Knol JC, Eichberger P, Schiestl B, Debbage P, Skvortsova I and Lukas P: Radioresistant head and neck squamous cell carcinoma cells: Intracellular signaling, putative biomarkers for tumor recurrences and possible therapeutic targets. Radiother Oncol 101: 177-182, 2011.

25. Chen PJ, Yeh SH, Liu WH, Lin CC, Huang HC, Chen CL, Chen DS and Chen PJ: Androgen pathway stimulates microRNA-216a transcription to suppress the tumor suppressor in lung cancer-1 gene in early hepatocarcinogenesis. Hepatology 56: 632-643, 2012.

26. Xia H, Ooi LL and Hui KM: MicroRNA-216a/217-induced epithelial-mesenchymal transition targets PTEN and SMAD7 to promote drug resistance and recurrence of liver cancer. Hepatology 58: 629-641, 2013.

27. Yu J, Li A, Hong SM, Hruban RH and Goggins M: MicroRNA alterations of pancreatic intraepithelial neoplasias. Clin Cancer Res 18: 981-992, 2012. 
28. Lomonaco SL, Finniss S, Xiang C, Decarvalho A, Umansky F, Kalkanis SN, Mikkelsen T and Brodie C: The induction of autophagy by gamma-radiation contributes to the radioresistance of glioma stem cells. Int J Cancer 125: 717-722, 2009.

29. Zhuang W, Qin Z and Liang Z: The role of autophagy in sensitizing malignant glioma cells to radiation therapy. Acta Biochim Biophys Sin (Shanghai) 41: 341-351, 2009.

30. He WS, Dai XF, Jin M, Liu CW and Rent JH: Hypoxia-induced autophagy confers resistance of breast cancer cells to ionizing radiation. Oncol Res 20: 251-258, 2012.

31. Chaachouay H, Ohneseit P, Toulany M, Kehlbach R, Multhoff G and Rodemann HP: Autophagy contributes to resistance of tumor cells to ionizing radiation. Radiother Oncol 99: 287-292, 2011.

32. Fujiwara K, Iwado E, Mills GB, Sawaya R, Kondo S and Kondo Y: Akt inhibitor shows anticancer and radiosensitizing effects in malignant glioma cells by inducing autophagy. Int J Oncol 31: 753-760, 2007.

33. Kim EJ, Jeong JH, Bae S, Kang S, Kim CH and Lim YB: mTOR inhibitors radiosensitize PTEN-deficient non-small-cell lung cancer cells harboring an EGFR activating mutation by inducing autophagy. J Cell Biochem 114: 1248-1256, 2013.

34. Cheng H, Li J, Liu C, Yao W, Xu Y, Frank TS, Cai X, Shi S, Lu Y, Qin Y, et al: Profilin1 sensitizes pancreatic cancer cells to irradiation by inducing apoptosis and reducing autophagy. Curr Mol Med 13: 1368-1375, 2013.
35. Wang P, Zhang J, Zhang L, Zhu Z, Fan J, Chen L, Zhuang L, Luo J, Chen H, Liu L et al: MicroRNA 23b regulates autophagy associated with radioresistance of pancreatic cancer cells. Gastroenterology 145: 1133-1143, 2013.

36. Menghini R, Casagrande V, Marino A, Marchetti V, Cardellini M, Stoehr R, Rizza S, Martelli E, Greco S, Mauriello A, et al: miR-216a: A link between endothelial dysfunction and autophagy. Cell Death Dis 5: e1029, 2014.

37. Zou Z, Wu L, Ding H, Wang Y, Zhang Y, Chen X, Chen X, Zhang CY, Zhang Q and Zen K: microRNA-30a sensitizes tumor cells to cis-platinum via suppressing beclin 1-mediated autophagy. J Biol Chem 287: 4148-4156, 2012.

38. Chatterjee A, Chattopadhyay D and Chakrabarti G: miR-17-5p downregulation contributes to paclitaxel resistance of lung cancer cells through altering beclin1 expression. PLoS One 9: e95716, 2014.

39. Chen Y, Li X, Wu X, He C, Guo L, Zhang S, Xiao Y, Guo W and Tan B: Autophagy-related proteins LC3 and beclin-1 impact the efficacy of chemoradiation on esophageal squamous cell carcinoma. Pathol Res Pract 209: 562-567, 2013. 